\title{
Surface Morphology of Polyphenylsilsesquioxanes/Hydroxyl- Functionalized Polystyrene Blends Investigated by Atomic Force Microscopy
}

\author{
Dong Wook KJm, Seung Sang Hwang, ${ }^{\dagger}$ Soon Man Hong, and Eung-Chan LeE \\ Hybrid Polymer Research Center, Korea Institute of Science and Technology, \\ P.O. Box 131, Cheongryang, Seoul 130-650, Korea
}

(Received July 19, 1999)

\begin{abstract}
Phase separation morphology in the thin blend film of polyphenylsilsesquioxane (PPSQ) and hydroxylfunctionalized polystyrene (PSOH) was investigated. $\mathrm{PSOH}$ was synthesized by anionic polymerization and identified with TLC, ${ }^{1} \mathrm{H}$ NMR, and GPC. The two polymers were dissolved in a common solvent and the thin film was prepared by spincoating. Atomic force microscopy (AFM) has been used to investigate the surface morphology of the thin blend films. In $20 \%$ PPSQ and $80 \%$ PSOH blends, the PPSQ-rich phase was uniformly dispersed like granules and domain size was as small as $100 \mathrm{~nm}$. Comparing it with the PPSQ/PS blend with the same content of PPSQ, the domain size is about one third of that of the PPSQ/PS blend. This enhanced miscibility is believed to result from intermolecular hydrogen bonding between the PPSQ phase and the PSOH phase, which was verified by IR investigation. However, the PPSQ/PSOH (80/ 20 ) blend showed morphology of the so large domain distributed irregularly. The size of dispersed phase ranged from 500 $\mathrm{nm}$ to $1500 \mathrm{~nm}$.
\end{abstract}

KEY WORDS Hydroxyl-Functionalized Polystyrene / Polyphenylsilsquioxanes / Atomic Force Microscopy / Phase Behavior / Surface Morphology /

Since the first commercialization by Owens Illinois under the name of Glass Resin, researchers have studied the synthesis, physical properties, and applications of polysilsesquioxanes. Particularly polyphenylsilsesquioxane (PPSQ) having a rigid ladder-like structure has attracted much attention due to excellent properties such as solubility in a common solvent, thermal stability, mechanical strength, and electrical insulation. The use of PPSQ has focused upon coatings in electronics and optical devices. In recent years the applications have ranged to interlayer dielectrics, photonic materials such as optical waveguide, gas separation membranes, and carcinostatic drugs. ${ }^{1-10}$

Some researchers have tried to broaden the application fields of PPSQ through blend or block copolymerization with organic polymers. Recently, the blend of PPSQ with polystyrene (PS) has been studied by Li et al. ${ }^{11} \mathrm{PS}$ and PPSQ in the blends prepared by the solution casting had the phases separated on a scale of several thousand nanometers. To enhance the miscibility, in situ blend was tried, but it was not effective due to the limited interaction between molecules of PS and PPSQ. There are several techniques to enhance interactions between phases in polymer blends. One of the most effective methods is to introduce functional groups into the polymers. $^{12-15}$

The surface morphology of a thin blend film is significantly different from the bulk morphology. The phase separation process is usually strongly influenced by the polymer-air interface. Moreover, during the spincoating process the solvent is rapidly evaporated in a very short time, leading to phase separation. In many typical applications of PPSQ such as coatings in electronics and optical devices, the polymer films are prepared by spincoating. Accordingly, study on the surface morphology of the

\footnotetext{
${ }^{\dagger}$ To whom correspondence should be addressed.
}

PPSQ-containing thin blend film is important to understand the phase separation and the polymer-air interface effects which determine wettability and adhesion, etc. Atomic force microscopy (AFM) has opened a new world for investigating the polymer surface morphology. Various force microscopy techniques enable us to measure phase separation on a nanometer scale. ${ }^{16--20}$ In this article we prepared a thin blend film of the PPSQ and the hydroxyl-functionalized polystyrene. The thin film was prepared by spincoating and then the surface morphology of the film was studied by AFM.

\section{EXPERIMENTAL}

\section{Synthesis of Polyphenylsilsesquioxanes}

Polyphenylsilsesquioxanes (PPSQ) were synthesized as previously. ${ }^{4-6}$ The synthetic routes and chemical structures are shown in Scheme 1.

\section{Synthesis of Hydroxyl-Functionalized Polystyrene}

Hydroxyl-functionalized polystyrene (PSOH) was prepared by anionic polymerization of the polystyrene followed by introduction of the hydroxyl group into the chain end using ethylene oxide as shown in Scheme 2.

Styrene and benzene were purified according to the literature. ${ }^{21} s$-Butyllithium (Aldrich Chem. Co., 1.7 M in pentane) was used as the initiator for polymerization without further purification. Ethylene oxide (Aldrich Chem. Co., 99.5\%) was stirred over calcium hydride. After several times degassing in the high vacuum line and distillation into a flask containing dibutyl magnesium, ethylene oxide was divided into ampoules followed by dilution with benzene. Polymerization was done in a sealed reactor using breakseals and a standard high vacuum technique. The formation of polymeric organolithium compounds could be readily identified by a specific orange-red color. After overnight reaction, ethylene ox- 
<smiles>Cl[Si](Cl)(Cl)c1ccccc1</smiles>

Trichlorophenylsilane (TCP)

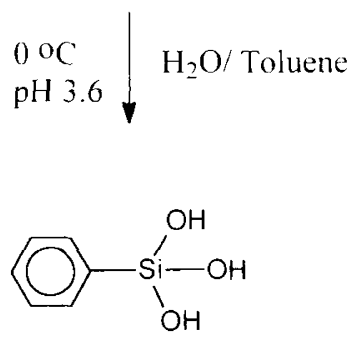

TCP hydrolysate

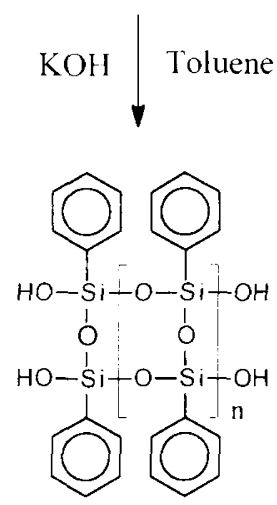

PPSQ

Scheme 1. Synthesis of polyphenylsilsesquioxanes.

ide in the ampoule was put into the reactor through a breakseal. The polystyryllithium compound was so active that the color of the solution readily faded away. To complete the reaction, additional stirring was allowed for a couple of hours, and the solution was put into excess methanol containing a small amount of aqueous HCl. To exclude impurities, the polymer powder was washed with methanol several times. ${ }^{1} \mathrm{H}$ NMR $\left(\mathrm{CDCl}_{3}\right)$; $\delta(\mathrm{ppm})=7.4-6.3$ (aromatic protons), $3.3\left(-\mathrm{CH}_{2} \mathrm{O}^{-}\right), 2.3$ -0.8 (backbone $\mathrm{CH}_{2}$ ).

\section{Characterization of Polymers}

Molecular weight and molecular weight distribution of the synthesized polymers were determined using a Waters GPC 410 system equipped with five Ultra- $\mu$ styragel columns performed at a flow rate of $1.0 \mathrm{~mL}$ $\min ^{-1}$ in THF. The values of $M_{n}, M_{w}$, and $M_{w} / M_{n}$ were calculated using a calibration curve generated with standard polystyrene samples. ${ }^{1} \mathrm{H}$ NMR spectra were obtained using a Varian Gemini-200 spectrometer. IR absorption spectra were measured on a Matson series 5000 .

\section{Preparation of the Thin Blend Films}

To study the morphology of the thin film, we prepared very dilute solutions with a common solvent. The $\mathrm{PSOH}$ and the PPSQ at different weight ratios were dissolved in toluene at a concentration of $0.8 \%$ by weight. The

$$
\text { sec }-\mathrm{C}_{4} \mathrm{H}_{9} \mathrm{Li}^{+}
$$

$+$

$\mathrm{m}$

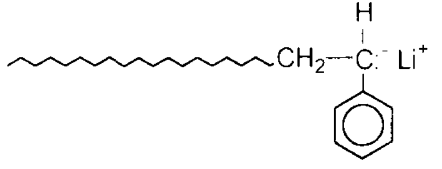

Ethylene oxide

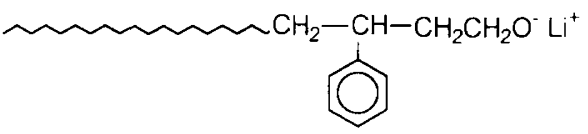

Methanol/ $\mathrm{HCl}$

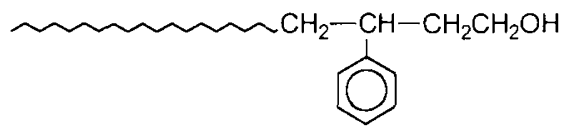

PSOH

Scheme 2. Synthesis of hydroxyl-functionalized polystyrene.

Table I. Compositions of specimens for PPSQ/PSOH blends

\begin{tabular}{cc}
\hline Sample No. & $\begin{array}{c}\text { Compositions of specimens } \\
\text { (Weight ratio) }\end{array}$ \\
\hline 1 & $\mathrm{PS}-\mathrm{OH} / \mathrm{PPSQ}=80 / 20$ \\
2 & $\mathrm{PS}-\mathrm{OH} / \mathrm{PPSQ}=50 / 50$ \\
3 & $\mathrm{PS}-\mathrm{OH} / \mathrm{PPSQ}=20 / 80$ \\
\hline
\end{tabular}

compositions of all samples are listed in Table I. The solution was spincoated onto a glass slide at $1000 \mathrm{rpm}$ after filtering the solution through a Millipore Teflon filter $(0.2 \mu \mathrm{m})$. The glass was washed with chloroform and acetone to clean out organic impurities and dried before use. No further treatment was done on the glass substrate. The average film thickness ranged from $80 \mathrm{~nm}$ to $150 \mathrm{~nm}$. The spincoated films were dried at $100^{\circ} \mathrm{C}$ for 24 $\mathrm{h}$ in the vacuum oven.

\section{AFM Measurement}

AFM images were taken with a Multimode Scanning Probe Microscope (Digital Instruments, Inc.). Tappingmode was used to get height imaging data with $125 \mu \mathrm{m}$ long cantilevers with a spring constant of $20-100 \mathrm{~N}$ $\mathrm{m}^{-1}$. The lateral scan frequency was about $1.0 \mathrm{~Hz}$. The sample was moved in the $x-y$ plane and a voltage was 


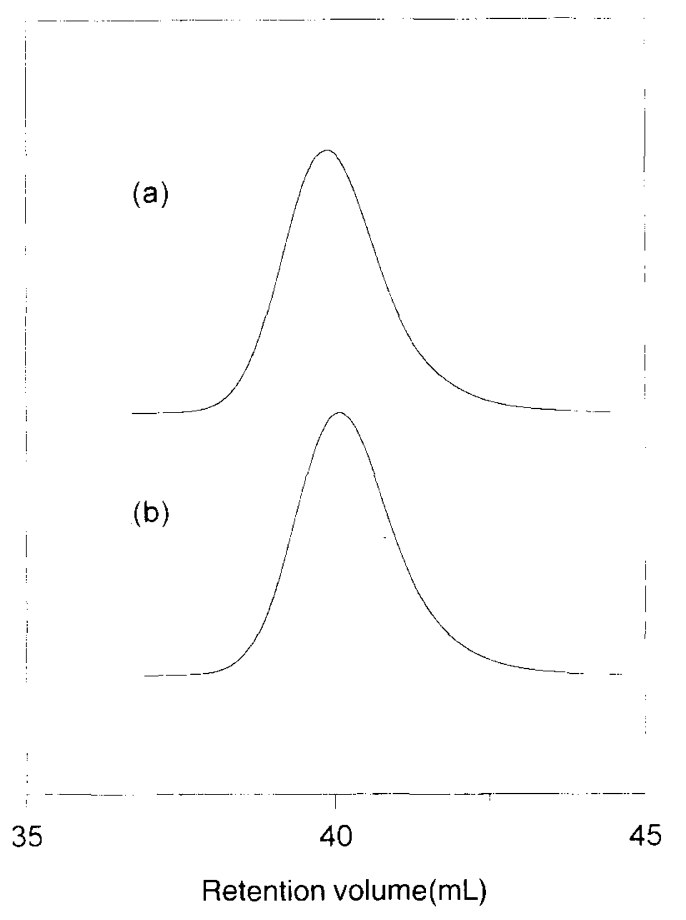

Figure 1. GPC chromatograms of (a) polystyrene and (b) hydroxyl-functionalized polystyrene.

applied, which moved the piezo driver over the $z$-axis, to keep the probing force constant, resulting in a three dimensional height image of the sample surface.

\section{RESULTS AND DISCUSSION}

\section{Preparation of Polymers}

PPSQ was synthesized as shown in Scheme 1. Trichlorophenylsilane was hydrolyzed in toluene/water mixture and then the hydrolysate was polymerized to the high molecular weight ladder structure in toluene with a small amount of potassium hydroxide. The chemical structure of the polymer was identified by ${ }^{29} \mathrm{Si} \mathrm{NMR},{ }^{1} \mathrm{H}$ NMR, wide angle X-ray scattering, and GPC, as described in detail previously. ${ }^{4-6}$ PPSQ has four hydroxyl groups in the chain end, which was proved by the distinct peak of hydroxyl proton at $1.5 \mathrm{ppm}$ and rather broad peak of phenyl proton at $6-8 \mathrm{ppm}$ in ${ }^{1} \mathrm{H}$ NMR spectrum. The molecular weight determined by ${ }^{1} \mathrm{H}$ NMR was 28000 .

Hydroxyl-functionalized polystyrene was synthesized by anionic polymerization in a high vacuum line. Polystyryllithium compound was prepared in a sealed reactor and reacted with ethylene oxide resulting in the endfunctional polystyrene. The product was identified by thin layer chromatography (TLC), ${ }^{1} \mathrm{H}$ NMR, FT-IR, and GPC. Figure 1 shows GPC chromatograms of the polystyrene and hydroxyl-functionalized polystyrene. The single narrow peak is apparent, which means that there are no coupling by-products. $M_{n}$ and $M_{w} / M_{n}$ of the PSOH were determined to be 5000 and 1.09 , respectively. Evidence that polystyryllithium was perfectly converted to hydroxyl-functionalized polystyrene was provided by TLC and ${ }^{1} \mathrm{H}$ NMR. From examination by TLC on silica gel plates no spot for unfunctionalized polymer was

Polym. J., Vol. 32, No. 7, 2000

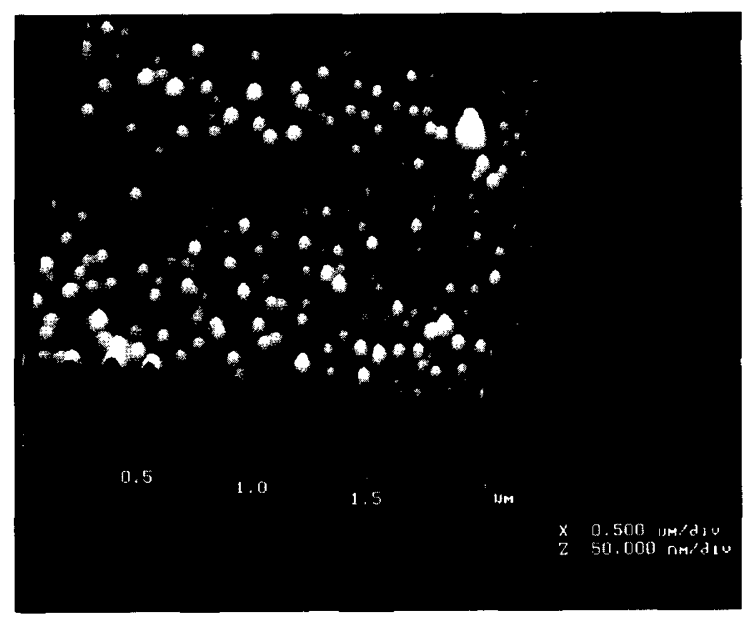

(a)

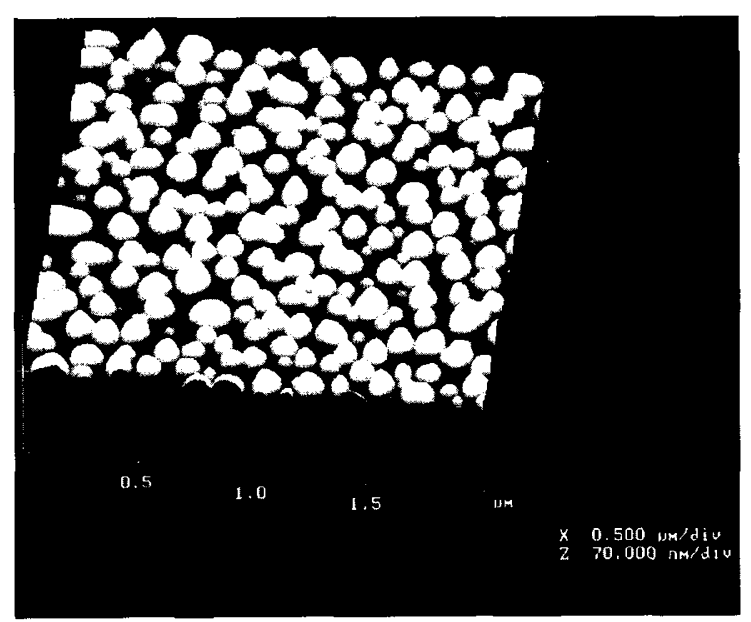

(b)

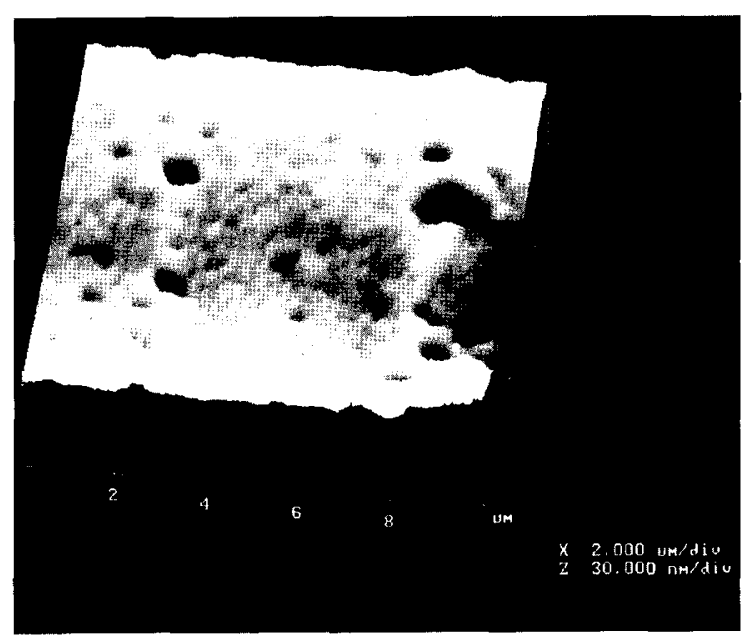

(c)

Figure 2. AFM images of PPSQ/PSOH thin blend films. (a) PPSQ/PSOH (20/80); (b) PPSQ/PSOH(50/50); (c) PPSQ/PSOH(80/20).

found. In ${ }^{1} \mathrm{H}$ NMR spectrum of PSOH, small broad single peak at $3.3 \mathrm{ppm}$ must be assigned to the methylene hydrogens bonded to the carbon, which is adjacent to the terminal hydroxyl group. When the ratio of the area of this peak to the area of the aromatic hydrogens was calculated, the molecular weight calculated from the value 
was in accord with that from GPC.

\section{Surface Morphology of the Thin Blend Films}

Figure 2 shows AFM images of PPSQ/PSOH blends. In the images of samples 1 and 2 (Figures $2 a$ and $2 b$ ) are granules distributed over the flat matrix with relatively regular size and uniform distribution. The size of domain ranged from $70 \mathrm{~nm}$ to $120 \mathrm{~nm}$ for the PPSQ/PSOH (20/80) blend and $70 \mathrm{~nm}$ to $210 \mathrm{~nm}$ for the PPSQ/PSOH (50/50) blend. Cavities are dispersed in the image of the PPSQ/PSOH (80/20) blend (Figure 2c). The domains are irregularly dispersed and size is quite large and variable, from $500 \mathrm{~nm}$ to $1500 \mathrm{~nm}$.

Since the surface morphology of the thin blend films is strongly influenced by interactions between the polymer surface and air, the surface energy of the blend components is important factor on the surface topography. In general, the polymer component with lower surface energy segregates preferentially to the air interface to minimize the polymer-air surface tension. ${ }^{22,23}$ PPSQ has lower surface energy relative to the PS $\left(27.7 \mathrm{mN} \mathrm{m}^{-1}\right.$ us. $\left.40.7 \mathrm{mN} \mathrm{m}^{-1}\right)^{1,24}$ and so the PPSQ-rich phase is supposed to protrude from the PS-rich one. Accordingly, the island consists of a PPSQ-rich phase, while the matrix consists of the PSOH-rich one in Figures $2 \mathrm{a}$ and $2 \mathrm{~b}$. Comparing the image of sample 1 with sample 2 , the increase of granules with content of PPSQ supports the assumption that the island is the PPSQ-rich phase. By the same reason, the PPSQ/PSOH (80/20) blend shows the morphology that the PPSQ-rich phase forms a continuous matrix, while the PSOH-rich one becomes a cavity.

The domains of samples 1 and 2 are distributed very uniformly and size is quite small. Considering the samples are just the blends not the block copolymer, the images are remarkable. For comparison, we prepared the thin blend film of the PPSQ and PS. PS has no hydroxyl group, and was synthesized by anionic polymerization. The molecular weight and polydispersity index were 10000 and 1.09, respectively. PPSQ and PS were dissolved in toluene with $0.8 \mathrm{wt} \%$. After spincoated onto a glass substrate with $1000 \mathrm{rpm}$, the film was dried in a vacuum oven at $100^{\circ} \mathrm{C}$ for $24 \mathrm{~h}$.

The AFM image of the PPSQ/PS (20/80) thin film is shown in Figure 3. Like the PPSQ/PSOH (20/80) blend, the PPSQ-rich phase forms an island on the PS-rich continuous phase. The image is similar to the one of sample 1 in Figure 2a. But the domain size is about 3 times larger than sample 1. The difference between the two samples is the hydroxyl end group. The enhanced miscibility of the PPSQ/PSOH blend may have been due to interfacial interactions between two phases such as the hydrogen bonding. FT-IR was used to examine possible interactions between the two components. The PPSQ/ PSOH blend solution and PPSQ/PS blend solution were cast on the $\mathrm{NaCl}$ crystal window for IR measurement and dried at $100^{\circ} \mathrm{C}$ in a vacuum oven, respectively. To avoid hydrogen bonding between the polymer and moisture in the air, FT-IR was measured immediately after the film was taken out of the oven.

Figure 4 shows the FT-IR spectra of the PPSQ/PSOH blend films. For comparison, IR data for the PSOH and PPSQ were also taken. A sharp and weak hydroxyl absorption band centered at $3600 \mathrm{~cm}^{-1}$ in Figure 4a indi-

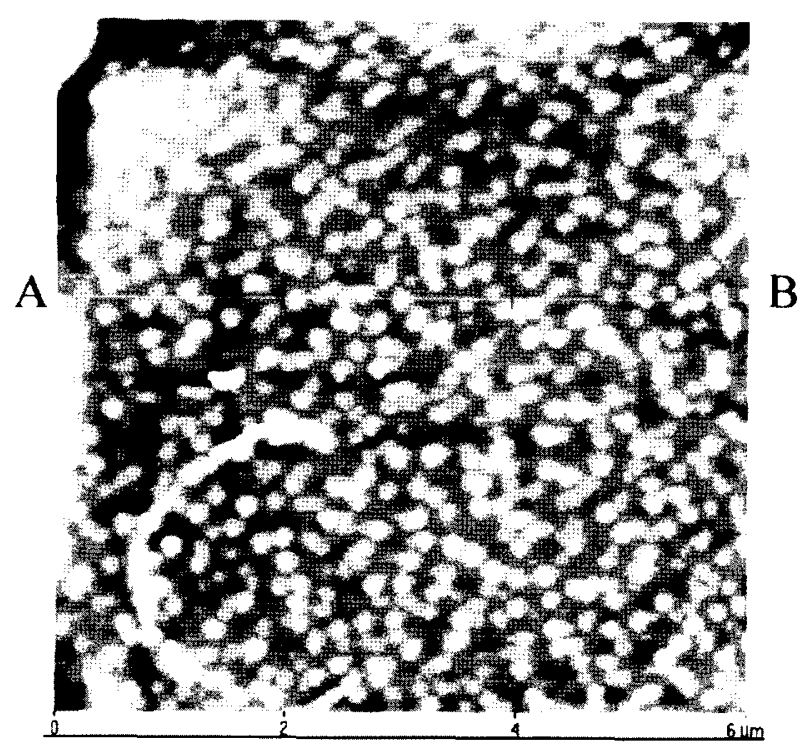

(a)

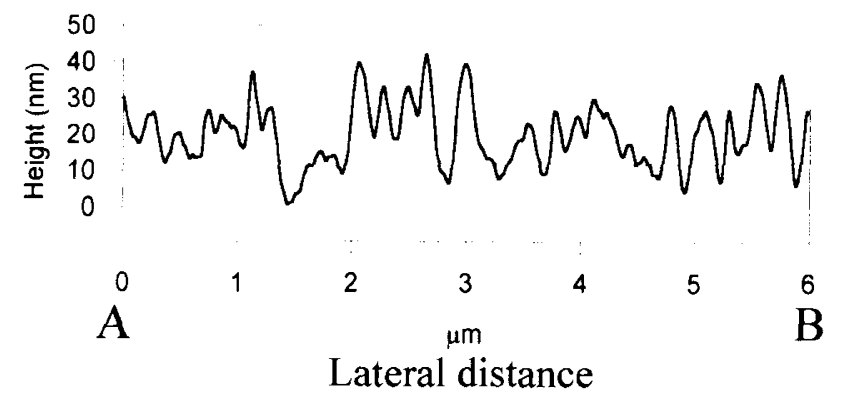

(b)

Figure 3. AFM image and sectional view of PPQS/PS(80/20) thin film. (a) AFM image; (b) sectional view along the line $(\mathrm{A}-\mathrm{B})$.

cates that the hydroxyl end groups in the PSOH film form little intermolecular hydrogen bonds within it. With increase in PPSQ in the PPSQ/PSOH blends, the broad absorption band centered at $3400 \mathrm{~cm}^{-1}$ newly appears and the intensity becomes stronger. This means the intermolecular hydrogen bonds formation. However, it is not certain whether the absorption band arises from the hydrogen bonding between PPSQ and PSOH, because PPSQ itself can form intermolecular hydrogen bonds as shown in Figure 4e.

To confirm this, we compared IR data of the PPSQ/ $\mathrm{PSOH}$ and PPSQ/PS blends (Figure 5). PPSQ/PS solution was cast onto the $\mathrm{NaCl}$ crystal window and dried at $100^{\circ} \mathrm{C}$ for $24 \mathrm{~h}$ in the vacuum oven before IR measuring. To compare the absorption intensity for PPSQ/PSOH films with PPSQ/PS films, we normalized the absorption intensity of the two blend films on the basis of the $\mathrm{C}-\mathrm{H}$ stretching absorption band, because the band should have the same intensity at the same content of PSOH or PS. From Figures 5a and 5b, it is certain that the PPSQ/ PS (20/80) blend has few hydrogen bonds, while the PPSQ/PSOH blend shows the broad band centered at $3430 \mathrm{~cm}^{-1}$ together with the unbonded or free hydroxyl band at $3610 \mathrm{~cm}^{-1}$. The difference is more obvious on comparing Figures $5 \mathrm{c}$ and $5 \mathrm{~d}$. This indicates that the broad hydroxyl bands centered at $3410 \mathrm{~cm}^{-1}$ in Figures $5 \mathrm{a}$ and $5 \mathrm{c}$ are due to intermolecular hydrogen bonds be- 


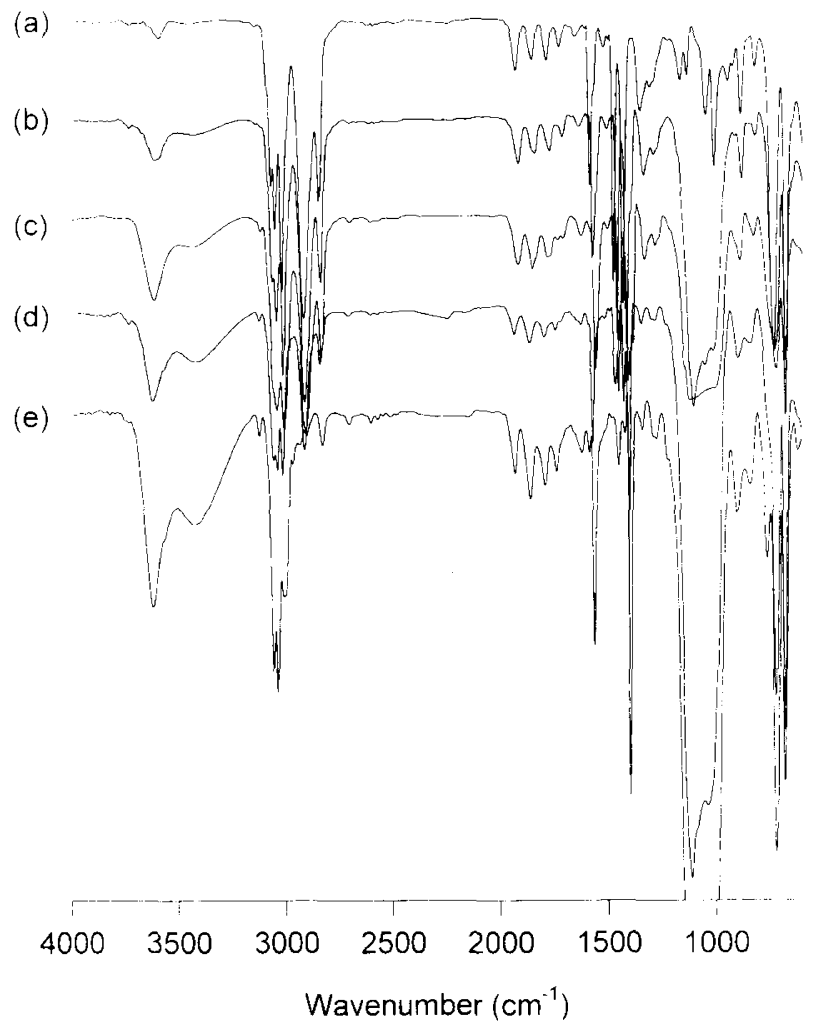

Figure 4. FT-IR spectra of PPSQ/PSOH blend films cast on the $\mathrm{NaCl}$ crystal window for IR measurement. (a) PSOH; (b) PPSQ/ PSOH (20/80); (c) PPSQ/PSOH (50/50); (d) PPSQ/PSOH (80/20); (e) PPSQ.

tween PPSQ and PSOH. Accordingly, it is assumed that the uniform distribution of the so small domain in the PPSQ/PSOH blends is attributed to intermolecular hydrogen bonds between PPSQ and PSOH phases.

As the content of PPSQ reaches $80 \%$, the PPSQ/PS blend shows a broad absorption band at $3425 \mathrm{~cm}^{-1}$ due to intermolecular hydrogen bonds within PPSQ itself. The hydroxyl band of the PPSQ/PSOH (80/20) has the same shape and intensity with the PPSQ/PS (80/20) as shown in Figures 5e and 5f. This means that, as the content of PPSQ becomes higher, PPSQ forms intermolecular hydrogen bonds preferentially with itself, not with PSOH. This may explain the AFM image of the PPSQ/ PSOH $(80 / 20)$ blend shown in Figure 2c. Since hydrogen bonds are few between PPSQ and PSOH phases, the domain is irregularly distributed and size is very large.

\section{CONCLUSIONS}

Introducing a hydroxyl group into the polystyrene was very effective to enhance interfacial interactions in the PPSQ/PS blend. Comparison of IR data of PPSQ/PSOH with PPSQ/PS blends verified that the intermolecular hydrogen bonds form between PPSQ and the PSOH phases. Hydrogen bonds between two components lead to the morphology that the very small size of domain is distributed regularly. The size of the domain is as small as $100 \mathrm{~nm}$ in the PPSQ/PSOH (20/80) thin blend film. However, in the case of PPSQ/PSOH (80/20) blend, the domain is irregularly distributed and size is quite large, probably because, as content of PPSQ becomes higher, (a)

(c)

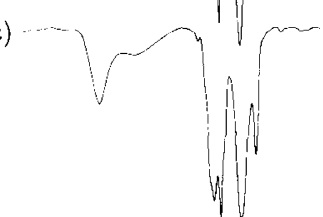

(e)
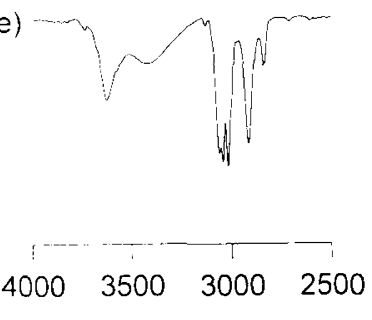

Wavenumber $\left(\mathrm{cm}^{-1}\right)$ (b)

(d)

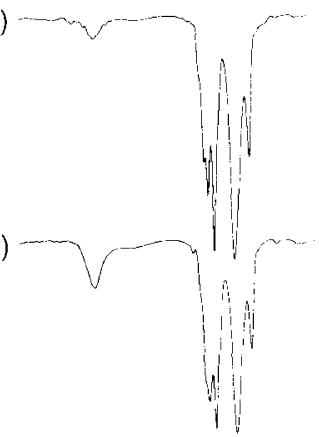

(f)

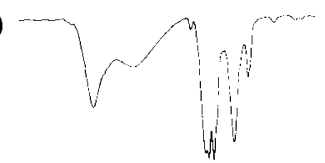

$4000 \quad 3500 \quad 3000 \quad 2500$

Wavenumber $\left(\mathrm{cm}^{-1}\right)$
Figure 5. FT-IR spectra in the region of $4000-2500 \mathrm{~cm}^{-1}$ of the blend thin films cast on the $\mathrm{NaCl}$ crystal window. (a) PPSQ/PSOH (20/80); (b) PPSQ/PS (20/80); (c) PPSQ/PSOH (50/50); (d) PPSQ/ PS (50/50); (e) PPSQ/PSOH (80/20); (f) PPSQ/PS (80/20).

PPSQ forms intermolecular hydrogen bonds preferentially with itself, not with PSOH.

\section{REFERENCES}

1. R. H. Baney, M. Itoh, A. Sakakibara, and T. Suzuki, Chem. Rev., 95, 1409 (1995).

2. D. A. Loy and K. J. Shea, Chem. Rev., 95, 1431 (1995).

3. O. V. Mukbaniani, V. A. Achelashvili, M. G. Karchkhadze, R. S. Tkeshelashvili, V. Y. Levin, and L. M. Khananashvili, Int. J. Polym. Mater., 18, 129 (1992).

4. E.-C. Lee and Y. Kimura, Polym. J., 29, 678 (1997).

5. E.-C. Lee and Y. Kimura, Polym. J., 30, 234 (1998).

6. E.-C. Lee and Y. Kimura, Polym. J., 30, 730 (1998).

7. Z. Li, X. Cao, H. Xu, P. Xie, M. Cao, and R. Zhang, React. Func. Polym., 39, 1 (1999).

8. D. A. Schneider, D. A. Loy, B. M. Baugher, D. R. Wheeler, R. A. Assink, T. M. Alam, and R. Saunder, Polym. Prepr., Am. Chem. Soc., Div. Polym. Chem., 39, 513 (1998).

9. H. Ni, A. D. Skaja, R. A. Sialer, and M. D. Soucek, Polym. Mater. Sci. Eng., 79, 21 (1998).

10. P. Xie and R. Zhang, Polym. Adv. Technol., 8, 649 (1997).

11. G. Li, Y. Zin, L. Shi, M. Ye, and F. Bai, J. Polym. Sci., Part B: Polym. Phys., 34, 1079 (1996).

12. M. M. Coleman, G. J. Pehlert, and P. C. Painter, Macromolecules, 29, 6820 (1996).

13. G. J. Pehlert, P. C. Painter, B. Vetysman, and M. M. Coleman, Macromolecules, 30, 3671 (1997).

14. M. M. Coleman and P. C. Painter, Macromol. Chem. Phys., 199, 1307 (1998).

15. A. R. Oromehie, S. A. Hashemi, I. G. Meldrum, and D. N. Waters, Polym. Int., 42, 117 (1997).

16. R. W. Carpick, Chem. Rev., 97, 1163 (1997).

17. R. S. McLean and B. B. Sauer, Macromolecules, 30, 8314 (1997).

18. H. G. Dikland, S. S. Sheiko, L. van der Does, M. Moller, and A. Bantjes, Polymer, 34, 1773 (1993).

19. D. Reifer, R. Windeit, R. J. Kumpf, A. Karbach, and H. Fuchs, Thin Solid Films, 264, 148 (1995).

20. L. Nick, A. Kindermann, and J. Fuhrmann, Colloid Polym. Sci, 272, 367 (1994).

21. R. P. Quirk and W.-C. Chen, Makromol. Chem., 183, 2071 
(1982).

22. S. Walheim, M. Böltau, J. Mlynek, G. Krausch, and U. Steiner, Macromolecules, 30, 4995 (1997).

23. K. Tanaka, A. Takahara, and T. Kajiyama, Macromolecules,
29, 3232 (1996).

24. J. Brandrup and E. H. Immergut, "Polymer Handbook", 3rd ed, John Wiley \& Sons, New York, N. Y., 1989. 